\title{
Compact sensor on the basis of the "silicon on isolator" waveguide structure for express detecting of ammonia solutions
}

\begin{abstract}
The optical method for control of concentration of liquid ammonia solutions on the basis of the heterodyne waveguide structure "silicon on insulator" is discussed. Construction of a quasi single-mode wide aperture optical waveguide with diffraction grating is discussed. The single mode operation is provided thanks to low optical losses of a fundamental mode and the increased losses of the higher modes because of existence of additional high-doping areas. Construction possesses the raised metrological characteristics. The experimental results of approbation of a method are given.
\end{abstract}

Keywords: optical sensor, detecting of ammonia solutions, optical waveguide, silicon on isolator, optical losses
Volume 4 Issue I - 2018

\author{
Masalskii NV \\ Scientific Research Institute of System Analysis, Russia
}

Correspondence: Masalskii NV, Scientific Research Institute of System Analysis, Russian Academy of Sciences, Moskva, Russia, Tel 8-499-I24-73-76, Email volkov@niisi.ras.ru

Received: February 16, 2018 | Published: February 20, 2018

\section{Introduction}

Now great interest to optical methods of detection of the liquid and gaseous substances especially harmful and dangerous to the person, for example, of ammonia is marked. One of the perspective directions of implementation of these methods is the thin-film SOI (silicon on insulator) technology, in particular, application of the waveguide structures as a sensor element. Interest in such devices is caused by a row of their advantages. The SOI waveguide structures thank to the properties (excellent optical characteristics, miniaturization, firmness in hostile environment) give qualitatively new opportunities for optical methods of detection of liquid and gaseous substances in a broad range of applications. In this paper the possibility of implementation of selective detection of liquid ammonia on the basis of the heterogeneous quasi single-mode SOI waveguide structures by means of a diffraction grating element is discussed.

\section{Construction sensor}

The functional diagram of a sensor element is provided on figure 1a. The coherent optical radiation from the laser is entered into a waveguide (2), extends in it and is exhausted from a wave guide by means of diffraction grating. In the presence of the researched component (7) there is a change of place of the radiated peak. This shift registers by a CCD-array (5). The prism (4) is used for support of a wide angle range the reradiated optical beam on boundary a waveguide - an integumentary layer. When testing when filling grooves of a diffraction grating with polar ammonia molecules the maximum of the angular reflected light distribution displaces for several angular minutes. The solid line schematically showed the course of optical radiation out of the waveguide component in the presence of the researched substance, by a dotted line without it. The focusing system isn't shown on Figure 1. For support of high metrological characteristics the wide-aperture heterogeneous waveguide structure which diagram is provided on Figure 1B is used. In this structure areas with high concentration of two types of the free charge carriers (electrons and holes) are in addition created. These additional areas provide small optical losses and unrepresentative single-mode behavior for a thin-film wide-aperture (over 10 microns) high-contrast SOI waveguide. High-doping $\mathrm{n}+$ and $\mathrm{p}+$ areas are designed to suppress modes of the higher orders which inevitably arise in the wide aperture waveguides. It attracts the sharp growth of parasitic losses. Width $\mathrm{Wn}+$ of the $\mathrm{n}+$ area is approximately equal to a half of a core width $\mathrm{W}_{0}$. Width $\mathrm{Wp}+$ of the $\mathrm{p}+$ area is approximately equal to $\mathrm{W}_{0} / 4$. Therefore, $\mathrm{Wn}+=2 \mathrm{Wp}+$. Such alternating construction provides the high level of modal selection, suppression of re-radiation through a waveguide lateral face, the general electro-neutrality of the waveguide structure.
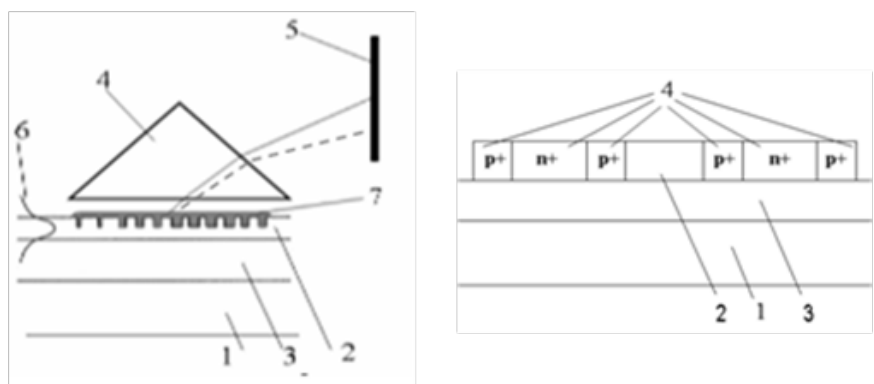

Figure I

IA: (Left) the sensor element diagram, where I - a silicon substrate, 2 - a silicon optical waveguide with diffraction grating, 3 - a burriex silicon oxide layer, 4 - a prism, 5 - a CCD-array, 6 - distribution of an optical mode in a waveguide, 7 - the researched substance.

IB: (Right) the diagram of SOI heterogeneous waveguide structure, where I - a silicon substrate, 2 - a silicon core, 3 - a thick $\mathrm{SiO}_{2}$ layer, 4 - high-doping areas.

Properties of a fundamental $\mathrm{m}_{0}$ - mode (Figure 2), except for smaller optical losses, in general are similar to properties of a zero mode of the standard semiconductor waveguide created at the expense of a gradient of the free charge carriers. Fundamental differences arise for all other modes $\left(\mathrm{m}_{1}, \mathrm{~m}_{2}, \ldots\right)$ a heterogeneous waveguide. Their effective refraction indexes are close or less refraction index of area 2 on Figure 1B. Their optical fields occupy all area of a waveguide. 
Therefore, the share of energy which falls on the dissipative area is many times more, than at a $\mathrm{m}_{0}$ mode (Figure 1B). These modes inevitably have the considerable additional attenuation on the free carriers which grows with number of a mode. These outputs are confirmed by results of simulation. Calculations were carried out by BMP and FDTD methods. By way of illustration, for example, it is shown that high selection of a fundamental mode is reached for an optical waveguide with the total aperture of 35 microns and $\mathrm{W}_{0}=11$ microns. At the same time its optical losses very low also make $-1.5 \mathrm{~dB}$ for the doping level is $9.0 \times 10^{17} \mathrm{~cm}^{3}$. Additional attenuation for the higher orders modes on the free charge carriers concerning the level of attenuation of a fundamental mode makes- $23.6 \mathrm{~dB}$ for $\mathrm{m}_{1}$ mode and-31.4 $\mathrm{dB}$ for $\mathrm{m}^{2}$ mode.

\section{Experimental results}

Choice and optimization of topology of the SOI waveguide structure with a diffraction grating explicitly are considered in. ${ }^{1}$ For structure with thickness: a waveguide - 210 nanometers, the burriex oxide - 940 nanometers, apertures: $\mathrm{W}_{0}-10.2$ microns, $\mathrm{Wn}+-5.1$ microns and $\mathrm{Wp}+-2.6$ microns, a doping concentration of $\mathrm{n}+$ and $\mathrm{p}+$ areas $-5 \times 10^{20} \mathrm{~cm}^{3}$, the groove minimum width is 22 nanometers and depth is 100 nanometers on wavelength of 1550 nanometers maximum

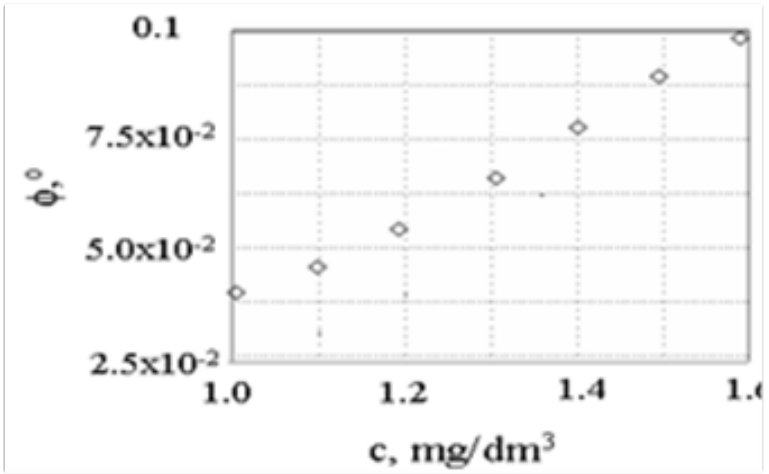

efficiency of an output is equal to $76 \%$. The experimental dependence is given in Figure 3A. It is important to mark that this shift can be watched easily, fixing angular provision of a peak of intensity, not resorting to spectral instruments. The temporal response of a sensor for different concentration of ammonia is given in Figure 3B.

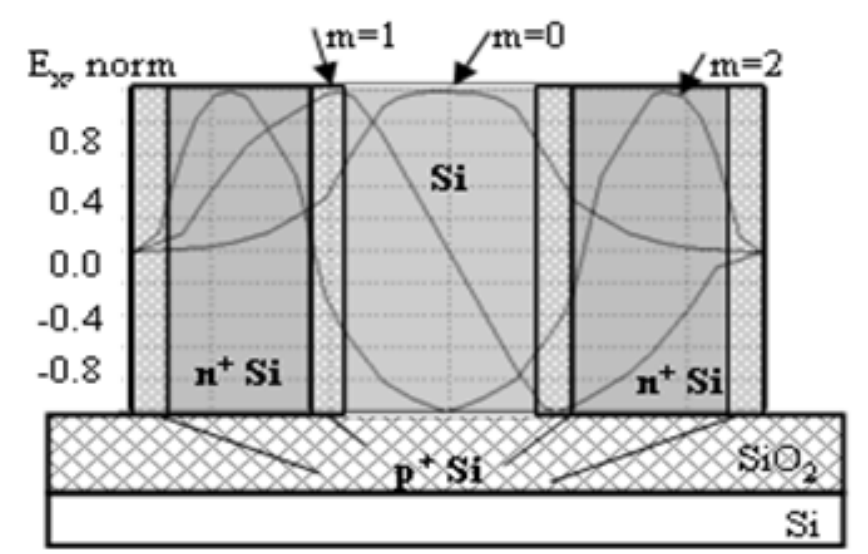

Figure 2 The normalized distribution of optical fields of the first three modes in case of $W_{0}=11$ microns.

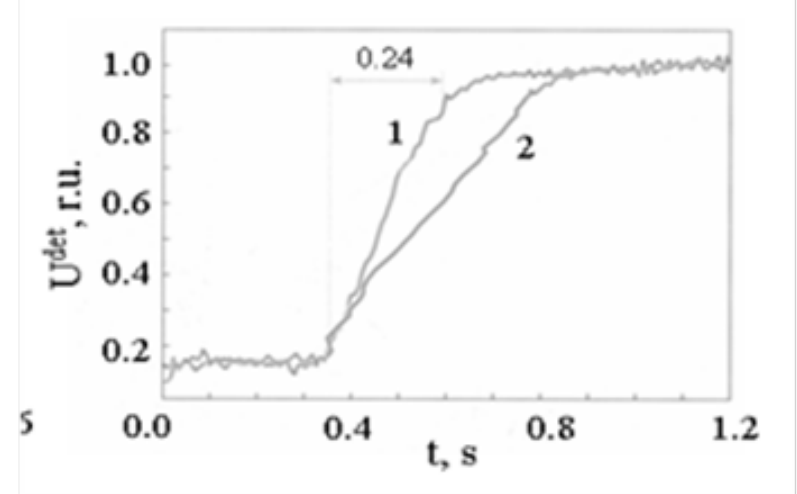

Figure 3

(A) (Left) the experimental dependence of angular displace of a peak of intensity on a logarithm of extremely low ammonia concentration.

(B) (Tight) the experimental temporal sensor response for different concentration where I- high, the average response time is $0.24 \mathrm{sec} ., 2-$ low, the average response time is $0.44 \mathrm{sec}$.

\section{Conclusion}

Construction of a compact optical sensor on the basis of the heterogeneous quasi single-mode SOI waveguide structure with diffraction grating is developed for monitoring of concentration of liquid ammonia solutions. Experimentally its characteristics for the low concentration solutions are probed. On condition of the subsequent computer processing the developed device shows excellent metrological characteristics.

\section{Conflict of interest}

The author declares no conflict of interest.

\section{References}

1. Taillaert D, Bienstman P, Baets R. Compact efficient broadband grating coupler for silicon-on-insulator waveguides. Optics Letters. 2004:29(23):2749-2751.

\section{Acknowledgements}

None. 